\title{
Editorial
}

\section{Interventional Endoscopy and Laparoscopy in GI Diseases}

In recent years we have witnessed a wide range of innovations and new developments in gastrointestinal endoscopy. New technical devices based on high magnification have further progressed to the dimension of microscopic endoscopy. Moreover, the visual assessment of the entire small bowel and the biliary/pancreatic system has become possible. These developments have revolutionized our approach to diseases of the gastrointestinal tract. The borders between traditional medical and surgical endoscopy are beginning to disappear, making room for more interaction across medical and surgical disciplines.

In this issue of Digestive Diseases we are introduced to the latest innovations in endoscopy and laparoscopy in gastrointestinal diseases. An international panel of highly skilled and renown surgical experts shares with us the advances in surgical endoscopy and laparoscopy in the major diseases of the gastrointestinal tract. Integrating gastroenterological and surgical points of view on these modern technologies will greatly benefit the treatment of our patients. In the end, we believe it really does not matter whether you are a gastroenterological surgeon or a surgical gastroenterologist, there is more common ground than we may believe today.

M.P. Ebert, Magdeburg

P. Malfertheiner, Magdeburg 